\title{
LONG-PERIOD MECHANISM OF THE 8 NOVEMBER 1980 EUREKA, CALIFORNIA, EARTHQUAKE
}

\author{
By Thorne Lay, Jefreey W. Given, and Hiroo Kanamori
}

\begin{abstract}
The seismic moment and source orientation of the 8 November 1980 Eureka, California, earthquake $\left(M_{s}=7.2\right)$ are determined using long-period surface and body wave data obtained from the SRO, ASRO, and IDA networks. The favorable azimuthal distribution of the recording stations allows a well-constrained mechanism to be determined by a simultaneous moment tensor inversion of the Love and Rayleigh wave observations. The shallow depth of the event precludes determination of the full moment tensor, but constraining $\boldsymbol{M}_{\mathbf{z x}}=\boldsymbol{M}_{\mathbf{z}}=\mathbf{0}$ and using a point source at 16-km depth gives a major double couple for period $T$ $=256 \mathrm{sec}$ with scalar moment $M_{0}=1.1 \cdot 10^{27}$ dyne-cm and a left-lateral vertical strike-slip orientation trending $\mathrm{N} 48.2^{\circ} \mathrm{E}$. The choice of fault planes is made on the basis of the aftershock distribution. This solution is insensitive to the depth of the point source for depths less than $33 \mathrm{~km}$. Using the moment tensor solution as a starting model, the Rayleigh and Love wave amplitude data alone are inverted in order to fine-tune the solution. This results in a slightly larger scalar moment of $1.28 \cdot 10^{27}$ dyne-cm, but insignificant $\left(<5^{\circ}\right)$ changes in strike and dip. The rake is not well enough resolved to indicate significant variation from the pure strike-slip solution. Additional amplitude inversions of the surface waves at periods ranging from 75 to $512 \mathrm{sec}$ yield a moment estimate of $1.3 \pm 0.2 \cdot 10^{27}$ dyne-cm, and a similar strike-slip fault orientation. The long-period $P$ and $S H$ waves recorded at SRO and ASRO stations are utilized to determine the seismic moment for 15- to 30-sec periods. A deconvolution algorithm developed by Kikuchi and Kanamori (1982) is used to determine the time function for the first $180 \mathrm{sec}$ of the $P$ and $\mathrm{SH}$ signals. The $\mathrm{SH}$ data are more stable and indicate a complex bilateral rupture with at least four subevents. The dominant first subevent has a moment of $6.4 \cdot 10^{26} \mathrm{dyne}-\mathrm{cm}$. Summing the moment of this and the next three subevents, all of which occur in the first $80 \mathrm{sec}$ of rupture, yields a moment of $1.3 \cdot 10^{27}$ dyne-cm. Thus, when the multiple source character of the body waves is taken into account, the seismic moment for the Eureka event throughout the period range 15 to $500 \mathrm{sec}$ is $1.3 \pm 0.2 \cdot 10^{27}$ dyne-cm.
\end{abstract}

\section{INTRODUCTION}

The 8 November 1980 earthquake which occurred offshore of Eureka, California, is the largest earthquake in California since the 1952 Kern County earthquake. The magnitudes of this event given in the Monthly Listing of the U.S. Geological Survey are $m_{b}=6.2, M_{L}=7.0(\mathrm{BRK})$, and $M_{S}=7.2$. From the long-period scalar moment determined in this paper, we find $M_{w}=7.3$, where $M_{w}$ is the magnitude determined from the seismic moment (Kanamori, 1977). The large size of this event makes it of particular interest for its tectonic implications.

Studies of the aftershocks of the Eureka earthquake show a lineation 120 to 140 $\mathrm{km}$ long trending $\mathrm{N} 50^{\circ} \mathrm{E}$, extending from the Mendocino fault to $60 \mathrm{~km}$ northwest of Eureka (Smith et al., 1981; Eaton, 1981). Focal mechanisms determined from stations in California and Oregon show left-lateral strike-slip solutions for the main shock and aftershocks (Smith et al., 1981; Eaton, 1981). This orientation of faulting suggests internal deformation of the Gorda plate, due in part to north-south compression in the vicinity of the Cape Mendocino triple junction. The northeastern 
orientation is consistent with the observations of topographic features in the Gorda Basin made by Silver (1971), and with the fault orientations of the 7 June $1975\left(M_{L}\right.$ $=5.2)$ and 26 November $1976\left(M_{S}=6.8\right)$ events determined by Smith (1976) and Smith et al. (1981). Previous large events in the region include the 31 January 1922 $M_{S}=7.3$ and 23 January $1923 M_{S}=7.2$ events.

In this paper, we utilize long-period surface waves to determine the source mechanism of the 1980 Eureka event. This is done by moment tensor inversion and fault model inversion of Rayleigh and Love wave recordings. The resulting focal mechanism and moment are well constrained and stable over a large range in period and prove consistent with the aftershock distribution and locally determined focal mechanisms. Body wave data are then utilized to determine the nature of strain release indicated by the signal complexity and to obtain a reliable moment estimate at periods of 10 to $30 \mathrm{sec}$.

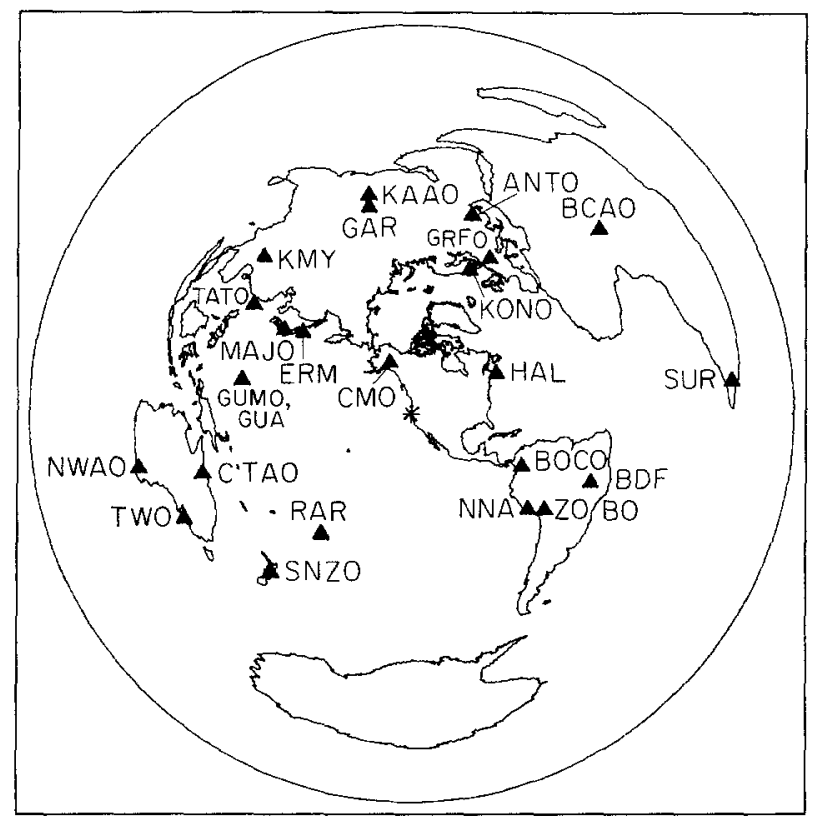

FIG. 1. Azimuthal equidistant plot centered on the 8 November 1980 Eureka, Calıforma, earthquake epicenter Locations of SRO, ASRO, and IDA stations used in this study are shown.

\section{DATA}

The three-component long-period recordings of the Eureka event made at Seismic Research Observatory (SRO) and Abbreviated Seismic Research Observatory (ASRO), and the long-period vertical components of the International Deployment of Accellerograph (IDA) stations provide our basic data set. Figure 1 shows the azimuthal distribution of these stations relative to the epicenter which is at $41.12^{\circ} \mathrm{N}$, $124.25^{\circ} \mathrm{W}$. The horizontal components were rotated into tangential and radial components, and all phases $R_{1}-R_{4}$ and $G_{1}-G_{4}$ were examined. The instrument calibration parameters showed the largest deviations from standard values for the horizontal components at ZOBO and KAAO, and the former were omitted in the surface wave analysis, while BOCO appears to have clipped for body wave arrivals.

For the purpose of obtaining a reliable long-period source mechanism, it is desirable to use the longest period for which the spectral behavior is stable. This enables one to have greater confidence in the use of gross earth phase velocity and 
$Q$ values in the inversion. Although well down on the amplitude response of the SRO and ASRO instruments, we found stable behavior for a period of $256 \mathrm{sec}$. For this period, similar inversion of Rayleigh wave data from the IDA network has proven successful (Kanamori and Given, 1981), and we adopted this period in all the initial processing. Many of the direct $R_{1}$ and $G_{1}$ arrivals were contaminated by nonlinear transients, and we chose to use principally $R_{2}, R_{3}, G_{2}$, and $G_{3}$ arrivals. All of the good quality data were equalized to the same propagational distance, and redundant pairs (such as $R_{2}$ and $R_{4}$ ) were checked to ensure that the amplitude and phase gave comparable results. This was found to be the case for all of the data, and gives us confidence in the stability of the signals at $256 \mathrm{sec}$. From the equalized data, we selected the phases indicated in Table 1 for the inversion procedure. In most cases, we could substitute $R_{4}$ or $G_{4}$ for $R_{2}$ or $G_{2}$ and have very little effect on the inversion.

TABLE 1

Stations Used in the Surface Wave Inversions

\begin{tabular}{lllrrl}
\hline Station Type & & \multicolumn{1}{c}{ Location } & $\Delta$ (deg) & Az (deg) & Phases Used \\
\hline ANTO & SRO & Ankara, Turkey & 96.5 & 17.5 & $R_{2}, R_{3}, G_{2}, G_{3}$ \\
BCAO & SRO & Bangu,, Central African Republic & 123.3 & 45.9 & $R_{1}, R_{2}, G_{2}, G_{3}$ \\
BOCO & SRO & Bogota, Columbia & 57.8 & 114.9 & $R_{2}, R_{3}, G_{2}, G_{3}$ \\
CTAO & ASRO & Charters Towers, Australıa & 102.6 & 254.5 & $R_{2}, R_{3}, G_{2}, G_{3}$ \\
GRFO & SRO & Grafenberg, Germany & 81.3 & 27.3 & $R_{3}, R_{4}, G_{1}, G_{2}$ \\
GUMO & SRO & Guam, Mariana Island & 81.8 & 281.0 & $R_{2}, R_{3}, G_{2}, G_{3}$ \\
KAAO & ASRO & Kabul, Afghanistan & 103.5 & 348.7 & $R_{2}, R_{3}, G_{2}, G_{3}$ \\
KONO & ASRO & Kongsberg, Norway & 72.5 & 225 & $R_{2}, R_{3}, G_{1}, G_{2}$ \\
MAJO & ASRO & Matsushiro, Japan & 71.3 & 303.0 & $R_{2}, R_{3}, G_{2}, G_{3}$ \\
NWAO & SRO & Narrogin, Australia & 131.1 & 2593 & $R_{2}, R_{3}, G_{2}, G_{3}$ \\
SNZO & SRO & South Karori, New Zealand & 99.1 & 2220 & $R_{2}, R_{3}, G_{2}, G_{3}$ \\
TATO & SRO & Taipei, Taiwan & 902 & 304.3 & $R_{2}, R_{3}, G_{1}, G_{2}$ \\
ZOBO & ASRO & Zongo Valley, Bolivia & 77.3 & 1250 & $R_{3}, R_{4}$ \\
BDF & IDA & Brasilia, Brazl & 90.4 & 110.4 & $R_{3}, R_{4}$ \\
CMO & IDA & College, Alaska & 27.3 & 338.2 & $R_{2}, R_{3}$ \\
ERM & IDA & Erimo, Japan & 65.6 & 3053 & $R_{2}, R_{3}$ \\
GAR & IDA & Garm, USSR & 98.9 & 348.5 & $R_{2}, R_{3}$ \\
GUA & IDA & Guam, Mariana Island & 818 & 2809 & $R_{2}, R_{3}$ \\
HAL & IDA & Halifax, Canada & 43.7 & 64.2 & $R_{3}$ \\
KMY & IDA & Kunming, PRC & 100.8 & 317.7 & $R_{1}, R_{2}$ \\
NNA & IDA & Naña, Peru & 68.7 & 129.2 & $R_{2}, R_{3}$ \\
RAR & IDA & Rarotonga, Cook Islands & 704 & 215.2 & $R_{2}, R_{3}$ \\
SUR & IDA & Sutherland, RSA & 150.9 & 83.1 & $R_{3}, R_{4}$ \\
TWO & IDA & Adelaide, Australia & 116.9 & 246.1 & $R_{2}, R_{3}$ \\
\hline
\end{tabular}

\section{INVERSION}

The inversion procedure used is that for a seismic moment tensor source described by Kanamori and Given (1981). This involves a least-squares inversion for the moment tensor under the assumption that the isotropic component is zero. It is clear from expression (14) in Kanamori and Given (1981) that by using Love wave data alone, one cannot determine all five elements of the moment tensor (the combination $M_{x x}+M_{y y}$ is not present), but combined with Rayleigh wave data the Love waves do provide additional constraint on the source orientation, particularly for strike-slip events.

Since it is reasonable to assume that the Eureka event is fairly shallow (USGS depth $=19.0 \mathrm{~km})$, there is a difficulty in determining the long-period source 
parameters. This is that the excitation functions $Q_{R}{ }^{(1)}$ (or $Q_{L}{ }^{(1)}$ for Love waves), in the notation of Kanamori and Stewart (1976), are derived from the radial factor of the stress function of normal modes which vanishes at the earth's surface. These excitation functions are coefficients of the $M_{z x}$ and $M_{z y}$ terms in the asymptotic expressions for the Rayleigh and Love displacement spectra, and hence those two elements of the moment tensor become indeterminate in the limit of zero depth. In order to stabilize the inversion for a shallow source, we follow the procedure discussed in Kanamori and Given (1981) of constraining $M_{z x}=M_{z y}=0$ in our inversions. This inherently restricts the solution space to either vertical strike-slip or $45^{\circ}$ dip-slip mechanisms. This restriction does not prove of major importance for the Eureka event, since it appears on the basis of the body and surface wave radiation patterns that the focal mechanism is indeed close to being a vertical strikeslip event. The excitation functions for the model 5.08 $M$ (Press, 1970; Kanamori, 1970), phase velocities computed from the normal mode data of Gilbert and Dziewonski (1975), and $Q$ and group velocity data from Kanamori (1970) are used in the inversion.

For the initial inversion, we used the Rayleigh wave data alone. We assumed a point source at $16-\mathrm{km}$ depth, and a source process time, $\tau=30 \mathrm{sec}$. The source process time accommodates phase shifts introduced by the source finiteness and finite source rise time. Since we lacked a priori knowledge of the rupture process, we chose the nondirectional value for $\tau$, where the overall source finiteness effect has the form

$$
\frac{\sin \left(\frac{\pi \tau}{T}\right)}{\left(\frac{\pi \tau}{T}\right)} \cdot \exp \left(\frac{-i \pi \tau}{T}\right)
$$

where $T$ is the period of the wave. For rupture lengths of up to $100 \mathrm{~km}$, this is a good approximation (Kanamori and Given, 1981). The choice of $\tau=30 \mathrm{sec}$ was based on the empirical relation given in Table 3 of Kanamori and Given (1981). The resulting moment tensor for the Rayleigh wave inversion is given in Table 2. The major double couple turns out to be the strike slip double couple with moment $1.0 \cdot 10^{27}$ dyne-cm and strike $\mathrm{N} 49.9^{\circ} \mathrm{E}$. The minor double couple has a moment 11 per cent the size of the major double-couple moment. The standard errors on the three moment tensor elements are quite small, indicating that this is a good solution. Figure 2 shows the azimuthal fit to the Rayleigh wave phase and amplitude spectra. The phase agreement is particularly good, indicating that the great circle paths for our source-receiver combinations are not unusually anomalous relative to the gross earth model. Since the inversion fits the complex signal, it is not surprising that the fit to the amplitude data is not as good as might be obtained by fitting amplitudes alone. The asymmetry in the theoretical radiation pattern is produced by the minor double couple, but it is clear that four lobes are present.

The next stage was combining the Love and Rayleigh wave data into a simultaneous inversion, noting that the Rayleigh wave solution would predict a four-lobed Love wave radiation pattern. The results of the simultaneous inversion with $\tau=30$ sec are given in Table 2, and the fit to the data is shown by the solid line in Figure 3. The solution is very similar to the previous one, with a minor change in strike and slight increase in moment. Two of the three moment tensor elements have lower standard deviations than the Rayleigh wave inversion. It is clear that the Love 
waves are quite consistent with a strike-slip solution. The four-lobed pattern is offset by $45^{\circ}$ from the Rayleigh wave pattern, and the nodes are well-constrained. Again, the phase agreement is very good, and the amplitudes are reasonably well fit, although the model appears to slightly underpredict the observations in the loop

TABLE 2

Moment TENSOR $(d=16 \mathrm{KM})$

CONSTRAINED $\left(M_{z x}=M_{z y}=0\right), \tau=30.0 \mathrm{SEC}$

\begin{tabular}{|c|c|c|}
\hline Data & Raylergh Waves & Rayleigh and Love Waves \\
\hline$M_{x y}{ }^{*}$ & $0.16 \pm 008$ & $011 \pm 007$ \\
\hline$M_{y y}-M_{x x}$ & $1.89 \pm 0.18$ & $2.03 \pm 013$ \\
\hline$M_{y y}+M_{x x}$ & $0.11 \pm 0.06$ & $0.11 \pm 009$ \\
\hline$M M_{z y}$ & 0 & 0 \\
\hline$M_{z x}$ & 0 & 0 \\
\hline$M_{0}^{*}$ & 1.02 & 1.07 \\
\hline Dip & $900^{\circ}$ & $90.0^{\circ}$ \\
\hline \multicolumn{3}{|l|}{ Major } \\
\hline \multicolumn{3}{|l|}{ D-C } \\
\hline Slip & $0.0^{\circ}$ & $00^{\circ}$ \\
\hline Strike & 49.9 & $48.2^{\circ}$ \\
\hline Mınor D-C & $11 \%$ & $10 \%$ \\
\hline
\end{tabular}

* Unit of moment tensor elements and scalar moment is $10^{27}$ dyme-cm.

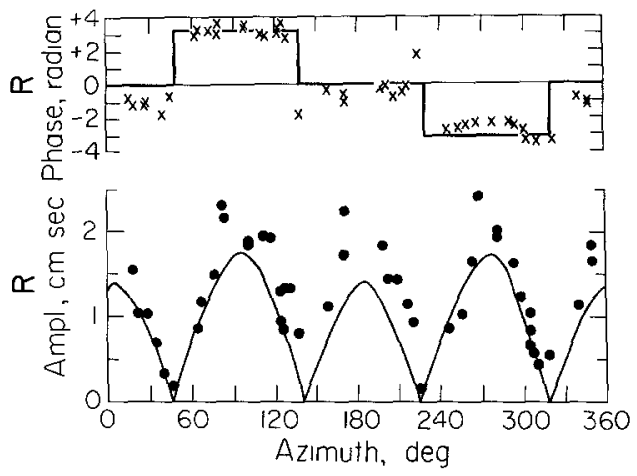

FIG 2. Phase and amplitude spectra of the 256-sec per1od Rayleigh waves of the Eureka earthquake. The phase spectra have been corrected for source finiteness using $\tau=30$ sec. The solid curves are for the Rayleigh wave inversion listed in Table 2.

directions. No asymmetry in the radiation patterns is evident, which suggests that directivity is not resolvable for this event at $256 \mathrm{sec}$. This is consistent with the relatively short fault length of 120 to $140 \mathrm{~km}$ inferred from the aftershock distribution.

Tests were conducted to assess the effect of the assumed source process time and point source depth. In Table 3, the moment tensor solutions of the simultaneous inversion for $\tau=0.0,30.0$, and $40.0 \mathrm{sec}$ are shown. The effects of varying the source process time are quite small, with a slight increase in moment and decrease in strike accompanying increasing $\tau$. The reduction in the minor double-couple contribution is more significant, and supports the selection of $\tau=30 \mathrm{sec}$. In Table 4, we show inversion solutions for varying source depths. The cases shown are for point sources at 9.75-, 16.0-, and 33.0-km depths and for excitation functions generated for a distributed source extending from 0 - to $24.5-\mathrm{km}$ depth (Kanamori and Given, 1981). 
The small changes in the resulting solutions indicate that there is no real sensitivity to depth in the 256-sec data despite the simultaneous use of Rayleigh and Love wave data. From these tests, we feel that the solutions given with $\tau=30$ sec and $d$
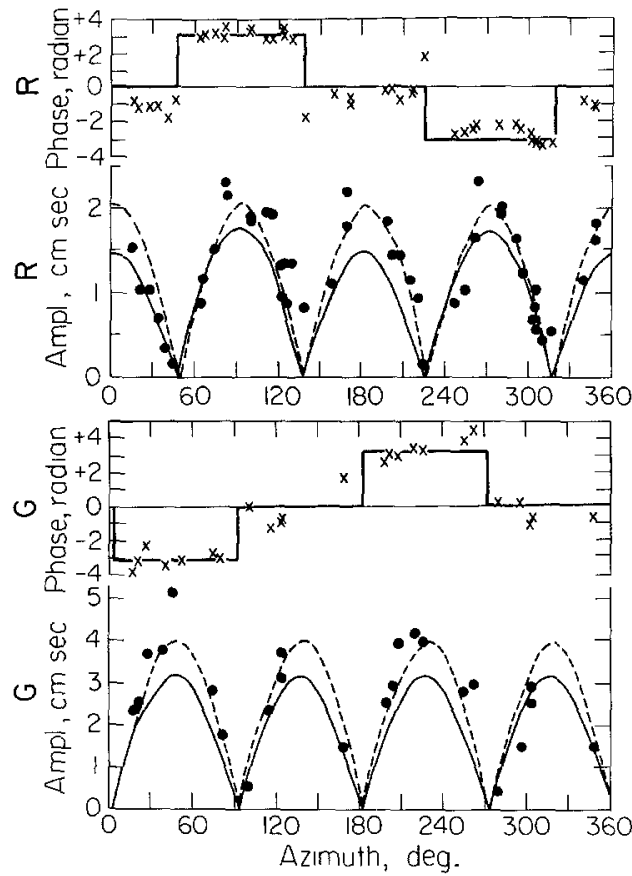

FIG. 3. Phase and amphtude spectra of the 256-sec period Rayleigh and Love waves of the Eureka earthquake. The phase spectra have been corrected for source finiteness using $\tau=30 \mathrm{sec}$. The solid curves are for the simultaneous Rayleigh and Love wave inversion listed in Table 2. The dashed lines are for the constrained-slip amplitude inversion in Table 5 .

TABLE 3

Moment TensoR $(d=16 \mathrm{KM})$

CONSTRAINED $\left(M_{z x}=M_{z y}=0\right)$

Simultaneous Rayleigh and Love Wave Inversion

\begin{tabular}{rcccc}
\hline & $\tau(\mathrm{sec})$ & 00 & 300 & 400 \\
\hline & $M_{x y}{ }^{*}$ & $014 \pm 0.08$ & $0.11 \pm 0.07$ & $0.08 \pm 0.07$ \\
& $M_{y y}-M_{x x}$ & $1.71 \pm 0.15$ & $2.03 \pm 0.13$ & $206 \pm 0.13$ \\
& $M_{y y}+M_{x x}$ & $0.22 \pm 0.10$ & $011 \pm 0.09$ & $0.06 \pm 0.09$ \\
& $M_{z y}$ & 0 & 0 & 0 \\
& $M_{z x}$ & 0 & 0 & 0 \\
& $M_{0}{ }^{*}$ & 098 & 1.07 & 1.07 \\
Major & Dip & $90.0^{\circ}$ & $90.0^{\circ}$ & $900^{\circ}$ \\
D-C & & & \\
& Shp & $0.0^{\circ}$ & $0.0^{\circ}$ & $0.0^{\circ}$ \\
Sinor D-C & $49.6^{\circ}$ & $48.2^{\circ}$ & $47.3^{\circ}$ \\
\hline
\end{tabular}

* Unit of moment tensor elements and scalar moment is $10^{27}$ dyne-cm.

$=16 \mathrm{~km}$ are as reliable as can be obtained from this data set. An attempt was made to relax the constraint on $M_{z x}$ and $M_{z y}$, but the inversion proved unstable.

Since the moment tensor inversion was constrained to be the vertical strike-slip or $45^{\circ}$ dip-slip solution and the fit to the amplitudes appeared biased toward slightly 
low values, we performed a nonlinear inversion using only the Love and Rayleigh wave amplitude data. This required specification of a starting model which was taken from the moment tensor result. This inversion allowed us to fine-tune the source mechanism and to seek any component of oblique slip. We performed the inversion for numerous starting models and combinations of constrained parameters. Some of the solutions are shown in Table 5. In all cases there was a tendency to increase the moment by 25 to 30 per cent, while the dip and strike fluctuated by

TABLE 4

Moment Tensor $(\tau=30 \mathrm{SeC})$

Constrained $\left(M_{z x}=M_{z y}=0\right)$

Simultaneous Rayleigh and Love Wave INVERsion

\begin{tabular}{cccccc}
\hline$d(\mathrm{~km})$ & 975 & 160 & 330 & $245 \mathrm{dm}^{*}$ \\
\hline & $M_{x y} \dagger$ & $009 \pm 0.07$ & $0.11 \pm 0.07$ & $0.12 \pm 0.07$ & $0.10 \pm 0.07$ \\
& $M_{y y}-M_{x x}$ & $1.97 \pm 013$ & $2.03 \pm 013$ & $2.10 \pm 0.14$ & $1.99 \pm 0.13$ \\
& $M_{y y}+M_{x x}$ & $009 \pm 0.08$ & $0.11 \pm 0.09$ & $0.15 \pm 0.11$ & $0.10 \pm 0.09$ \\
& $M_{z y}$ & 0 & 0 & 0 & 0 \\
& $M_{z x}$ & 0 & 0 & 0 & 0 \\
& $M_{0} \dagger$ & 1.03 & 107 & 113 & 1.05 \\
Major & Dip & $90.0^{\circ}$ & $90.0^{\circ}$ & $90.0^{\circ}$ & $90.0^{\circ}$ \\
D-C & & & & \\
& Slip & $0.0^{\circ}$ & $0.0^{\circ}$ & $0.0^{\circ}$ & $0.0^{\circ}$ \\
& Strike & $47.7^{\circ}$ & $48.2^{\circ}$ & $48.2^{\circ}$ & $47.8^{\circ}$ \\
Minor D-C & $9 \%$ & $10 \%$ & $13 \%$ & $9 \%$ \\
\hline
\end{tabular}

* Excitation function for distributed source extending from the surface to depth $24.5 \mathrm{~km}$.

$\dagger$ Unit of moment tensor elements and scalar moment is $10^{2 i}$ dyne-cm

TABLE 5

Amplitude InVERsion $(\tau=30$ SeC)

Simultaneous Rayleigh and Love Wave Inversion

\begin{tabular}{|c|c|c|c|c|}
\hline & $\begin{array}{c}\text { Startıng } \\
\text { Model }\end{array}$ & $\begin{array}{c}\text { Strike } \\
\text { Constramed }\end{array}$ & $\begin{array}{c}\text { Slup } \\
\text { Constrained }\end{array}$ & Unconstramed \\
\hline$M_{0}^{*}$ & 1.0 & 1.38 & 1.28 & 1.40 \\
\hline Dip & $900^{\circ}$ & $86.3^{\circ}$ & $90.0^{\circ}$ & $88.6^{\circ}$ \\
\hline Slpp & $00^{\circ}$ & $-25.0^{\circ}$ & $0.0^{\circ}$ & $-259^{\circ}$ \\
\hline Strike & $470^{\circ}$ & $48.0^{\circ}$ & $493^{\circ}$ & $48.6^{\circ}$ \\
\hline \multirow[t]{2}{*}{ S.E. } & & 0.469 & 0.452 & 0.463 \\
\hline & $\begin{array}{l}\text { Startung } \\
\text { Model }\end{array}$ & Unconstrained & $\begin{array}{l}\text { Starting } \\
\text { Model }\end{array}$ & Unconstramed \\
\hline$M_{0}^{*}$ & 1.0 & 128 & 1.0 & 1.28 \\
\hline Dup & $90.0^{\circ}$ & $98.7^{\circ}$ & $90.0^{\circ}$ & $79.6^{\circ}$ \\
\hline Slip & $10.0^{\circ}$ & $4.5^{\circ}$ & $-100^{\circ}$ & $-3.8^{\circ}$ \\
\hline Strike & $47.2^{\circ}$ & $48.6^{\circ}$ & $47.2^{\circ}$ & $48.6^{\circ}$ \\
\hline S.E. & & 0.464 & & 0.464 \\
\hline
\end{tabular}

* Unit of scalar moment is $10^{27}$ dyne-cm

only $\pm 5^{\circ}$. The rake, however was not stably resolved, varying with starting model, but never substantially reducing the standard error of the fit. Given this lack of constraint, the most reasonable solution appears to be for a rake of $0^{\circ}$, and a moment of $M_{0}=1.3 \cdot 10^{27}$ dyne-cm. The lack of variation in the strike and dip testifies to the degree to which the Love and Rayleigh wave data pin down the nodes of the radiation pattern. This result suggests that the constraint on the $M_{z x}$ and $M_{z y}$ components in the moment tensor inversion did not bias the solution. The 
fit of the rake-constrained amplitude inversion to the observations for a 16-km-deep point source and a 30-sec source process time is shown with a dashed line in Figure 3. The amplitude data are in better overall agreement with this model.

As a check on the solution obtained by the inversion, we calculated Love and Rayleigh wave synthetics for comparison with the data. Although good agreement does not conclusively prove that our mechanism is the correct one, the comparisons shown in Figures 4 and 5 illustrate the quality of the data and the solution. The synthetics in Figures 4 and 5 were calculated for the mechanism listed in Table 9 assuming a source process time of $30 \mathrm{sec}$. The numbers given in the figures are the moments observed at the individual station.

Most of the Love waves in Figure 4 were band-passed filtered between 80 and 1500 sec. The rest of the Love waves and the Rayleigh waves in Figure 5 were

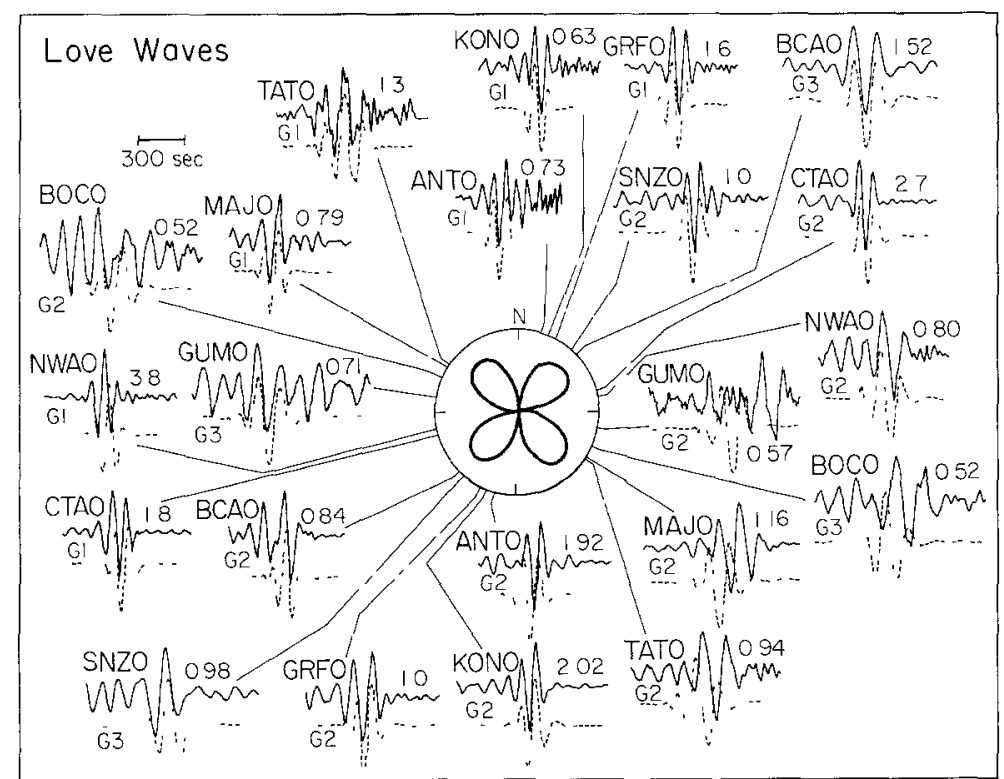

FIG. 4 The Love wave radiation pattern and comparison between synthetic (dotted lines) and observed seismograms. The fault parameters used in the synthetic calculation are listed in Table 9 , and a source process time of $30 \mathrm{sec}$ was assumed. The observed seismograms and synthetics for GUMO G2, GUMO $\mathrm{G}_{3}$, BOCO $\mathrm{G}_{2}$, BOCO $\mathrm{G}_{3}$, TATO $\mathrm{G}_{2}$, TATO $\mathrm{G}_{3}, \mathrm{MAJO}_{2}$, and MAJO $\mathrm{G}_{3}$ were filtered with a Gaussian band-pass filter between 120 and $1500 \mathrm{sec}$ The rest of the data and synthetics were band-pass filtered between 80 and $1500 \mathrm{sec}$. The number above each observed record is the moment (n units of $10^{27}$ dyne-cm) that would be obtained using that record alone.

filtered between 120 and 1500 sec. Because the SRO and ASRO instruments peak at around $30 \mathrm{sec}$, the seismograms are essentially narrow-band records of displacement. The dominant period is the short-period cut-off of the Gaussian band-pass filter. Therefore, our synthetic calculations provide a check of the inversion results at around $100 \mathrm{sec}$. The average moment obtained from the Love waves in Figure 4 is $1.27 \cdot 10^{27}$ dyne-cm, and from the Rayleigh waves in Figure 5, $1.7 \cdot 10^{27}$ dyne-cm. This result is in good agreement with the moment obtained from the 256 -sec period inversion. From Figures 4 and 5, it is apparent that the Love and Rayleigh wave amplitudes scatter significantly more at around $100 \mathrm{sec}$ than at $256 \mathrm{sec}$. This can be attributed to lateral heterogeneity and the inadequacy of the gross earth model used in the synthetic calculations. No systematic scatter which can be attributed to error 
in the mechanism is evident. Overall, the agreement between observed and synthetic seismograms is quite good and lends confidence to the inversion solution.

We applied the inversion procedure described above for several additional periods, ranging from 75 to $512 \mathrm{sec}$. For periods less than $150 \mathrm{sec}$, the use of gross earth model values for phase velocity and $Q$ introduces significant errors in the moment tensor inversion, while at periods longer than $300 \mathrm{sec}$ the low amplitudes and limited dynamic range of the instruments cause some spectral instability. Throughout the period range considered, reasonable amplitude behavior was observed, with phase inconsistency proving a greater problem. The results for constrained moment tensor and amplitude inversions are given in Tables 6 and 7. The moment tensor results are most reliable for periods greater than $200 \mathrm{sec}$, and the amplitude inversions are

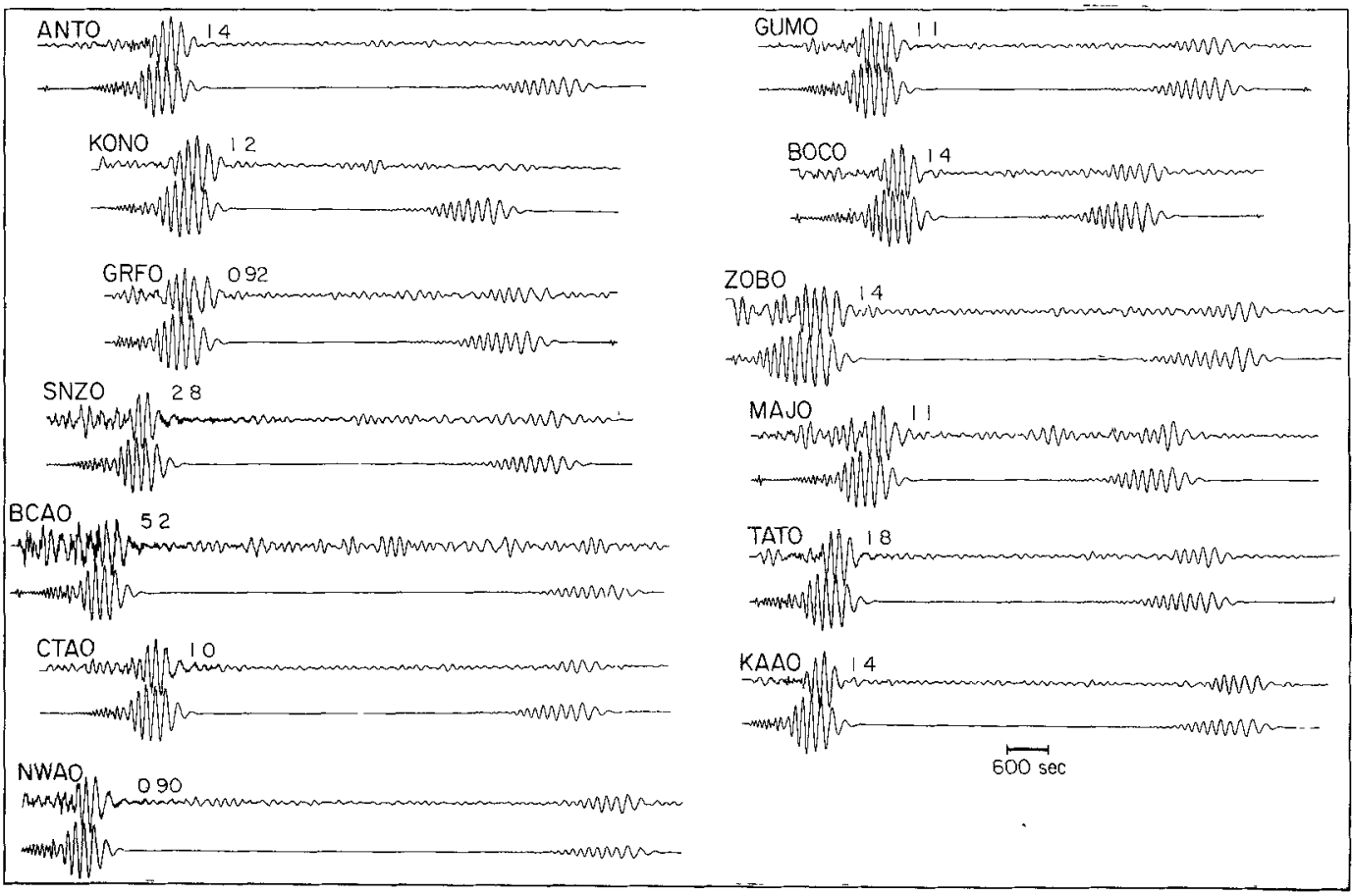

Fig. 5 Comparison between filtered Rayleigh wave synthetics and observed seismograms. The records are of $R_{2}$ and $R_{3}$ filtered in the pass-band 120 to 1500 sec The fault parameters used are the same as in Figure 4. The number above each observed record is the moment (in units of $10^{27}$ dyne-cm) that would be obtained using that record alone.

most reliable for periods from 128 to $301 \mathrm{sec}$. Over this period range, the amplitude inversions give a stable moment estimate of $1.26 \pm 0.09 \cdot 10^{27}$ dyne-cm. Shorter periods show much greater amplitude scatter as indicated by the increased standard error of the inversion solution, and the very long-period results are somewhat suspect simply because these periods are poorly excited and extremely far down on the instrument response. Despite these potential problems, the very long-period data show relatively small amplitude and phase scatter, and suggest a slight increase in moment with period. There is however, greater variation in strike at these periods and we do not feel that this trend is reliably resolved.

It is surprising that the amplitude inversion was successful for the 75-sec period. 
Lateral variations strongly affect such short periods, as does source directivity. For this case alone, the source process time was set to zero.

\section{Body Wave ANalysis}

The long-period $P$ and $S H$ waves recorded at the SRO and ASRO stations were modeled in order to obtain the seismic moment in the period range 10 to $30 \mathrm{sec}$ and

TABLE 6

Moment Tensor Inversions ( $\tau=30 \mathrm{Sec})$

Constrained $\left(M_{z x}=M_{z y}=0\right)$

Simultaneous Rayleigh and Love Wave Inversion

\begin{tabular}{|c|c|c|c|c|c|c|}
\hline$T$ (sec) & $M_{\mathrm{ry}}$ & $M_{n}-M_{x x}$ & $M_{y v}+M_{x x}$ & $M_{0}$ & $\begin{array}{l}\text { Strike } \\
\left({ }^{\circ}\right)\end{array}$ & $\begin{array}{c}\text { Minor D-C } \\
(\%)\end{array}$ \\
\hline 98.5 & $-0.05 \pm 0.12$ & $1.02 \pm 0.25$ & $-0.07 \pm 0.17$ & 0.5 & 42.5 & 13 \\
\hline 128.0 & $0.08 \pm 0.12$ & $0.88 \pm 0.26$ & $0.21 \pm 0.17$ & 0.6 & 50.1 & 38 \\
\hline 150.6 & $-0.08 \pm 0.12$ & $1.55 \pm 0.24$ & $0.08 \pm 0.16$ & 0.8 & 44.7 & 9 \\
\hline 176.6 & $0.00 \pm 0.08$ & $1.11 \pm 0.17$ & $0.07 \pm 0.11$ & 0.6 & 44.9 & 11 \\
\hline 196.9 & $0.19 \pm 0.09$ & $1.39 \pm 0.18$ & $0.01 \pm 0.12$ & 0.7 & 52.5 & 2 \\
\hline 222.6 & $0.10 \pm 0.08$ & $1.89 \pm 015$ & $0.06 \pm 0.10$ & 1.0 & 48.0 & 7 \\
\hline 256.0 & $0.11 \pm 0.07$ & $2.03 \pm 0.13$ & $0.11 \pm 0.09$ & 1.1 & 48.2 & 10 \\
\hline 301.2 & $0.20 \pm 0.07$ & $2.28 \pm 0.13$ & $0.00 \pm 0.09$ & 1.2 & 50.0 & 0 \\
\hline 341.3 & $0.42 \pm 0.08$ & $2.48 \pm 0.14$ & $-0.05 \pm 0.12$ & 1.3 & 54.3 & 4 \\
\hline 393.9 & $0.43 \pm 0.10$ & $2.53 \pm 0.17$ & $-0.05 \pm 0.17$ & 1.4 & 54.4 & 4 \\
\hline 465.5 & $0.26 \pm 0.11$ & $2.59 \pm 0.19$ & $-0.16 \pm 0.20$ & 1.4 & 50.6 & 11 \\
\hline 512.0 & $0.47 \pm 0.10$ & $2.27 \pm 0.19$ & $0.08 \pm 0.20$ & 1.3 & 56.2 & 7 \\
\hline
\end{tabular}

* Unit of moment tensor elements and scalar moment is $10^{27}$ dyne-cm.

TABLE 7

Amplitude InVERsions $(\tau=30 \mathrm{SEC})$

CONSTRAINED (DIP $\left.=90^{\circ}, \mathrm{SLIP}=0^{\circ}\right)$

Simultaneous Rayleigh and Love Wave Inversion

\begin{tabular}{cccc}
$T$ (sec) & $M^{*}{ }^{*}$ & $\begin{array}{c}\text { Strke } \\
\left({ }^{\circ}\right)\end{array}$ & $\mathrm{S} \mathrm{E}$ \\
\hline $75.3 \dagger$ & 1.32 & 50.9 & 1.8 \\
98.5 & 1.16 & 52.2 & 1.4 \\
1280 & 1.26 & 507 & 1.0 \\
150.6 & 1.34 & 49.4 & 08 \\
1766 & 1.10 & 499 & 0.7 \\
196.9 & 1.22 & 49.7 & 0.7 \\
222.6 & 127 & 499 & 0.6 \\
2560 & 1.28 & 49.3 & 0.5 \\
3912 & 1.35 & 51.4 & 0.5 \\
341.3 & 1.48 & 54.1 & 0.5 \\
393.9 & 1.54 & 525 & 0.6 \\
4655 & 151 & 50.2 & 0.7 \\
51.2 .0 & 1.44 & 52.5 & 0.6 \\
\hline
\end{tabular}

* Unit of scalar moment is $10^{27}$ dyne-cm.

$\dagger \tau=00 \mathrm{sec}$.

to investigate the mode of strain release of the Eureka earthquake. The long-period system response of the SRO stations has a peak response at $28-\mathrm{sec}$ period, with a 1.25-octave separation at $-3 \mathrm{~dB}$ points, and a $24-\mathrm{dB} /$ octave roll-off at high and low frequencies. In addition, there is a 6 -sec notch filter, and an antialiasing filter (McCowan and Lacoss, 1978). The net result is that the direct body wave phases 
observed on the long-period channels are relatively insensitive to the interference of direct and depth phases that is distinctive for shallow strike-slip events observed at WWSSN stations. Since these arrivals are not clearly distinguishable, it is difficult to constrain the focal mechanism further using this body wave data set, however the long-period nature of these signals does allow for a stable estimate of the moment, given independent knowledge of the source orientation. We employ the technique developed by Kikuchi and Kanamori (1982) to determine the body wave time function and moment. This technique involves a deconvolution procedure in which the far-field time function is obtained as a superposition of ramp functions. The height and onset time of the ramp functions are determined by matching the synthetic and observed waveforms in a least-squares sense. Discrete trapezoidal pulses produced by pairs of ramp functions are then identified as individual subevents, for both single- and multi-station deconvolutions.

The agreement between the surface wave fault orientation and the regional shortperiod mechanisms and aftershock distribution indicates that the fault orientation is well determined and not period dependent. In the deconvolution process, we fix the orientation to that of the surface wave results for the simultaneous inversion given in Table 2. The inversion procedure constrains all subevents to have the same orientation, which is a reasonable approximation except for nodal stations.

Figure 6a shows the azimuthal distribution of long-period $P$ waves along with the surface wave fault orientation. Many stations are very close to $P$-wave nodes, and the initial pulse is dominated by $s P$ which is predicted to be twice as large as $P$, and has the same polarity. $p P$ is of equal amplitude to $P$ and of opposite polarity which tends to cancel out the $P$ arrival for this shallow source. The depth phases all arrive within the 10-sec pulse width of the instrument impulse response. We inspected the short-period vertical channels to assure that the polarity of the $P$ arrival is consistent with that inferred on the long periods. The short-period data support an initial source depth of $19 \mathrm{~km}$ as given by the USGS, but it is not clear how this varies with time into the signal. Note that the signals are quite coherent at different azimuths and large amplitude arrivals are seen in the coda. Since $P c P$ is the only additional arrival predicted at these distances (and it should be small relative to the $P$ arrival), it is likely that the source is rather complex at these periods. This complexity is typical of that observed for $P$ waves from strike-slip events (e.g., Kanamori and Stewart, 1978), however, one must be cautious in drawing this conclusion, for the $P$ waves are all essentially nodal. The relative amplitudes show fairly stable behavior except for the stations nearest to $P$-wave nodes, where the amplitudes are enhanced.

The $S H$ data are shown in Figure $6 \mathrm{~b}$, and perhaps suggest a less complicated rupture process. Except at very nodal stations, KAAO and GUMO, the first pulse is clearly dominant. The polarities again prove consistent with the focal mechanism as do the relative amplitudes. This consistency was also observed in the $S V$ signals. For the $S H$ arrival, the phase $s S$ constructively interferes with the direct arrival for the given focal mechanism, and there is less rapid variation in their relative behavior than in the $P$-wave signal for small changes in fault orientation. This, combined with the less nodal character of the $S H$ phases leads us to place greater confidence in the $S H$ moment obtained below. There is some contamination due to $S c S$ in these signals, but again, this phase should be relatively small.

We first performed the deconvolution of each $P$ and $S H$ arrival individually. The individual time functions were then inspected for coherence of major arrivals and for variation in moment. We took 190-sec time windows except for a few closer stations where $170-\mathrm{sec}$ windows were adopted to avoid PP. The deconvolution 
procedure was allowed to run 40 iterations, with there being very little significant change after 30 iterations. The residual error given by equation (17) in Kikuchi and Kanamori (1982) was used to adjust the inversion parameters. We allowed only the depth to vary in the inversion procedure and found that the results are quite insensitive to source depth, but convergence is most rapid for a source depth of 5 $\mathrm{km}$. The ramp function rise time was also allowed to vary and was found to maximize the convergence with a value of $4 \mathrm{sec}$. In Figure 7 we show the resulting time functions, where a linear trend has been removed from each time function. This

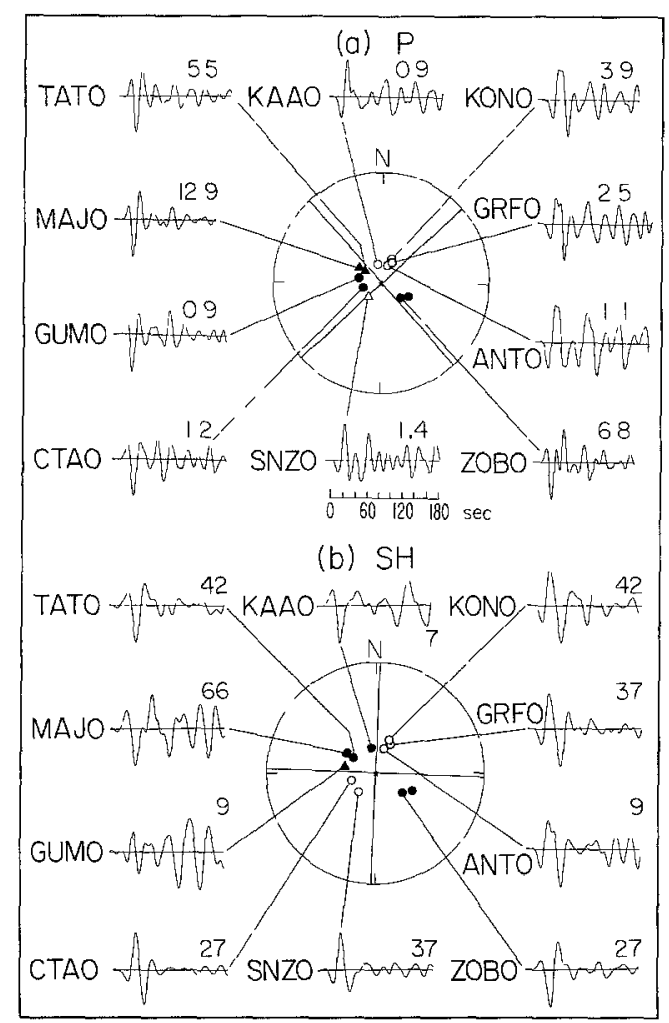

FIG. 6. (a) Long-period $P$-wave data from the SRO and ASRO networks along with the furst motions projected in the lower equal area hemisphere of the $P$-wave radiation pattern for the surface wave solution from the combined inversion in Table 2 Filled symbols denote compressional first arrivals and triangles indicate nodal stations (b) Long-perıod $S H$-wave data from the SRO and ASRO networks along with the $S H$ first motions projected in the lower equal area hemisphere of the $S H$-wave radiation pattern for the same solution as in (a). Filled symbols denote counterclockwise polarization relative to the source and triangles indicate nodal stations. In both figures, the numbers are the first peak-to-trough amplitudes in units of $10^{4}$ digital counts. ASRO stations have been corrected to SRO gains (5000 counts/micrometer of ground motion@25-sec period) for comparison

linear trend is probably caused by the low signal-to-noise ratio at the longer periods, and is not significant. Figure 8 , a and b, shows the synthetic $P$ and $S H$ waveforms corresponding to these time functions. The agreement in waveform is quite satisfactory, encouraging us to inspect the time functions in Figure 7 more closely.

In general, the $P$-wave time functions show greater structure than do the $S H$ results. Along with each time function, the corresponding moment taken from the first 120 to $140 \mathrm{sec}$ of the signal is given in Figure 7. The $P$-wave moments show a considerably larger moment range of a factor of 10 than do the $S H$ waves which show a factor of 2.2 . Note that the stations near nodes in the radiation pattern show 
consistently large moments and additional source complexity. The amplitudes at these stations are considerably larger than predicted by the surface wave solution. This behavior is often observed for nodal stations and in part may result from errors in the predicted radiation pattern, from changes in the radiation pattern with time, or from heterogeneity in the source region. The stations which show enhanced $P$ wave moments do not show corresponding $S H$ enhancement, nor are the surface wave amplitudes anomalous. We examined the WWSSN station MAT, which is near MAJO and found that the $P$ wave was similarly enhanced, although the initial arrival is very nodal as expected. The short periods at MAJO and ZOBO show that

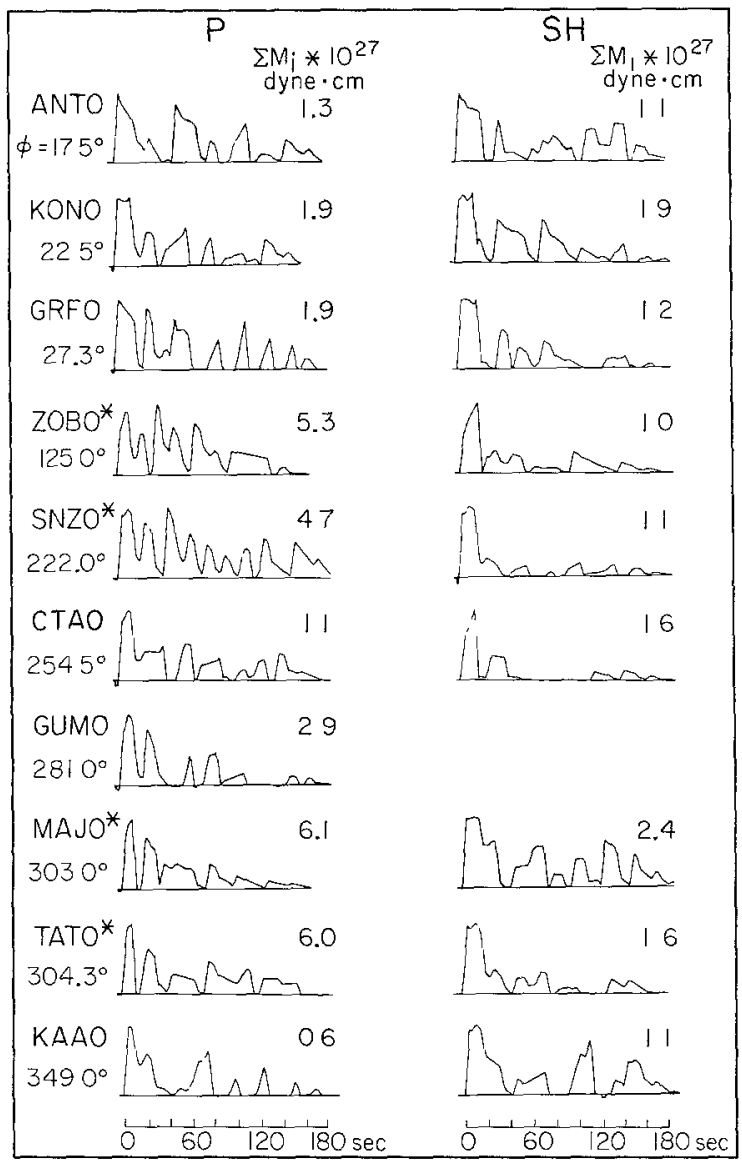

Frg 7 Individual station source time functions resulting from $P$ and $S H$ deconvolutions. The moments are taken from the first 120 to $140 \mathrm{sec}$ of each trace. Stations marked by an asterisk are very close to $P$-wave radiation nodes.

the initial $P$ arrivals are smaller than the coda in the following 30 sec and the absolute amplitudes of the first arrival are small relative to the other stations. This indicates that the fault orientation is quite accurate and that small changes in orientation probably occurred as rupture progressed. These changes most profoundly affect stations very close to nodes, and should affect the $S H$ waves less. For the simultaneous deconvolution we utilize only the six $P$ waves that are in more stable portions of the radiation patterns and use all of the $S H$ waves except for GUMO. 
The individual station time functions show fairly good coherence at particular azimuths, and rather substantial azimuthal variations. A consistent feature is that there are two fairly large events in the first $40 \mathrm{sec}$, although these are not as well resolved in the $S H$ waves as in the $P$ waves. This double source character was found to be the case in some preliminary forward modeling experiments. There appear to be three or four subsequent events, although these are not well resolved at some stations. Given the rather complete azimuthal coverage of this data set, the results are quite encouraging and justify the multi-station deconvolution which helps to suppress noise and core-reflected phases. It should be noted that the azimuthal coverage is much greater in this case than was available for the Guatemala earth-

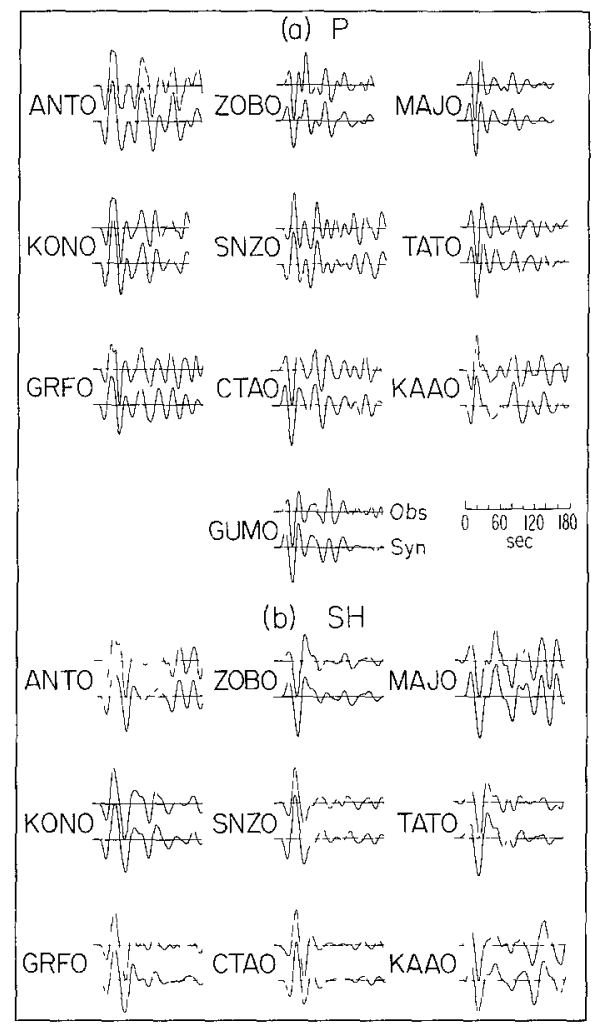

FIG. 8. (a) Comparison of observed and synthetic $P$ waves for the individual $P$-wave time functions in Figure 7. Each comparison is plotted with true relative amplitude. (b) Comparison of observed and synthetic $S H$ waves for the individual $S H$-wave tıme functions in Figure 7. Each comparison is plotted with true relative amplitude.

quake analyzed in Kikuchi and Kanamori (1982), and the variation in resulting individual time functions is correspondingly greater.

For the multi-station inversion, we take 11 points, each $15 \mathrm{~km}$ apart, along the fault strike as the discrete source locations, and invert the $P$ and $S H$ data separately. The far-field source time functions for the $P$ and $S H$ data evaluated at an azimuth $90^{\circ}$ from the fault strike are shown in Figure 9. The resulting synthetic waveforms are shown in Figure 10, a and b. The source time functions are encouragingly similar, with perhaps six discrete common sources in the first 160 sec. The moments of these ruptures are given in Table 8 . Nearly $\frac{1}{2}$ of the moment release occurs within the first $20 \mathrm{sec}$, but the additional ruptures do radiate significant energy. The $P$ and $S H$ time 

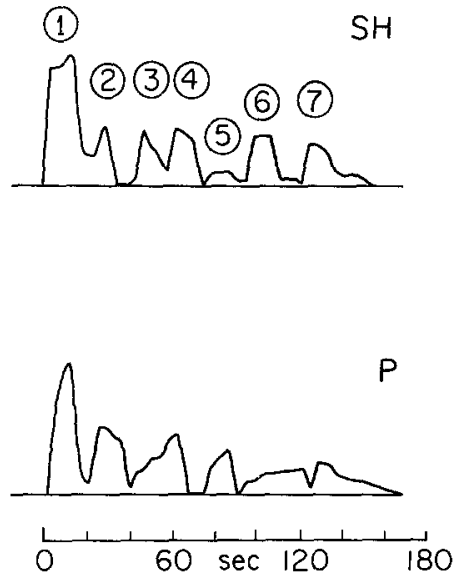

F1G. 9. $P$ and $S H$ time functions resulting from simultaneous deconvolutions. Discrete subevents are indicated with the circled numbers.

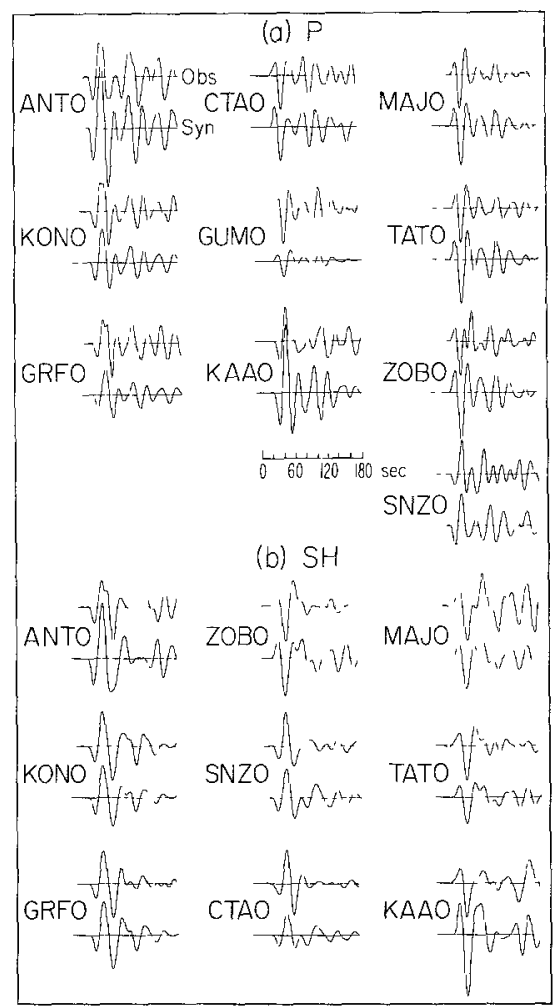

FIG. 10. (a) Comparison of observed and synthetic $P$ waves for the simultaneous $P$-wave deconvolution time function given in Figure 9. Each comparison is plotted with true relative amplitude. MAJO, TATO, ZOBO, and SNZO amplitudes have been reduced by a factor of 5.0. The latter stations were not used in the deconvolution. (b) Comparison of observed and synthetic $S H$ waves for the simultaneous $S H$ wave deconvolution time function given in Figure 9. Each comparison is plotted with true relative amplitude.

functions are similar in duration, although the initial pulse is 2 sec broader for the $S H$ waves.

In Figure 10a, the $P$-wave synthetics are compared with the data with true relative amplitudes. In addition to the six data used in the inversion we show comparisons 
for the additional four nodal stations where the observed amplitudes have been reduced by a factor of 5.0. The waveform agreement is quite good at most stations, although KAAO, which is a slightly diffracted arrival, is poorly fit. The misfit in amplitude of the synthetics tracks the range in moments found by the individual station deconvolutions shown in Figure 7. It is interesting that the waveform agreement at MAJO, TATO, ZOBO, and SNZO is reasonably good although these were not used in the inversion. The factor of 5 discrepancy in amplitude of these stations is apparently not associated with strong waveform characteristics. This is confirmed by inspecting the synthetics when the rake, dip, and strike are allowed to have $5^{\circ}$ variations. While this produces significant changes in predicted amplitude of up to a factor of 2 to 3 at nodal stations, the waveforms of the long periods are quite similar. Due to this waveform insensitivity, the SRO and ASRO data alone are too sparse to reliably fine-tune the focal mechanism. The simultaneous inversion result is quite stable for such small changes in orientation. This sensitivity of nodal stations indicates that caution must be exercised in using small sets of teleseismic $P$ waves to model source characteristics of strike-slip events.

The synthetic $S H$ waves are in quite good agreement with the data in both

TABLE 8

Moment and Process Time of Individual Subevents

\begin{tabular}{llccccccc}
\hline Phase Event & 1 & 2 & 3 & 4 & 5 & 6 & Total $^{*}$ \\
\hline \multirow{2}{*}{$P$} & $\tau(\mathrm{sec})$ & 11 & 15 & 14 & 9 & 11 & 30 & 49 \\
& $M_{0} \dagger$ & 61 & 4.1 & 2.0 & 2.0 & 2.0 & 2.6 & 14.2 \\
\multirow{3}{*}{$\mathrm{SH}$} & $\tau$ (sec) & 15 & 15 & 14 & 12 & 12 & 12 & 56 \\
& $M_{0} \dagger$ & 64 & 2.3 & 19 & 2.2 & 0.7 & 1.9 & 12.8 \\
\hline
\end{tabular}

* Taken for the furst four subevents.

+ Moment in $10^{26}$ dyne-cm.

TABLE 9

EUrEkA, 1980

Long-Period SOURCE MEchanism

\begin{tabular}{cccc}
\hline Moment (dyne-cm) & Strike & Dip & Shp \\
\hline $1.3 \pm 02 \cdot 10^{27}$ & $\mathrm{~N} 49^{\circ} \pm 02^{\circ} \mathbf{E}$ & $90^{\circ}$ & $0^{\circ}$ \\
\hline
\end{tabular}

waveform and relative amplitude. KAAO is again overpredicted, but the overall scatter is significantly less than found for the $P$ waves. Much of the complex interference at MAJO and TA'TO is modeled adequately, although the observations at CTAO and SNZO are simpler than the model. The greater stability of the $S H$ amplitudes suggests that the moment is best estimated as $1.3 \cdot 10^{27}$ dyne-cm, where we have taken the moment from the first $80 \mathrm{sec}$ of the $S H$ time function. This duration was chosen because it is that for which the $P$ and $S H$ results are most consistent and also proved more stable for small changes in fault strike.

The spatial variation of the moment release can be inferred from the time sequence of the discrete source activity, as shown in Figure 11. The solutions for both $P$ and $S H$ waves indicate a bilateral rupture which covered $75 \mathrm{~km}$ of the fault in the first $15 \mathrm{sec}$ of rupture. This indicates a rupture velocity of $2.5 \mathrm{~km} / \mathrm{sec}$. The next $20 \mathrm{sec}$ of rupture extended the rupture length at both ends of the fault as well as rupturing a "locked" spot in the middle. The rupture velocity and rupture 
duration are consistent with the 30 -sec source process time adopted in the surface wave analysis. It should be noted that the migration of aftershock activity was generally in a direction toward the southwest (Smith et al., 1981), which appears to contradict the bilateral pattern indicated in Figure 11. The complex interference of the subevents makes it difficult to resolve their spatial distribution, thus the evidence for bilateral rupture should be considered of marginal significance.

\section{Discussion}

For a moment of $1.3 \cdot 10^{27}$ dyne-cm one finds $M_{w}=7.3$, where

$$
M_{w}=\left(\log M_{0} / 1.5\right)-10.7 .
$$

This value of $M_{w}$ is slightly greater than the $M_{S}$ given by the USGS. If we assume a rigidity of $3 \cdot 10^{11} \mathrm{dyne} / \mathrm{cm}^{2}$, a fault length of $140 \mathrm{~km}$ and a width of $15 \mathrm{~km}$, we
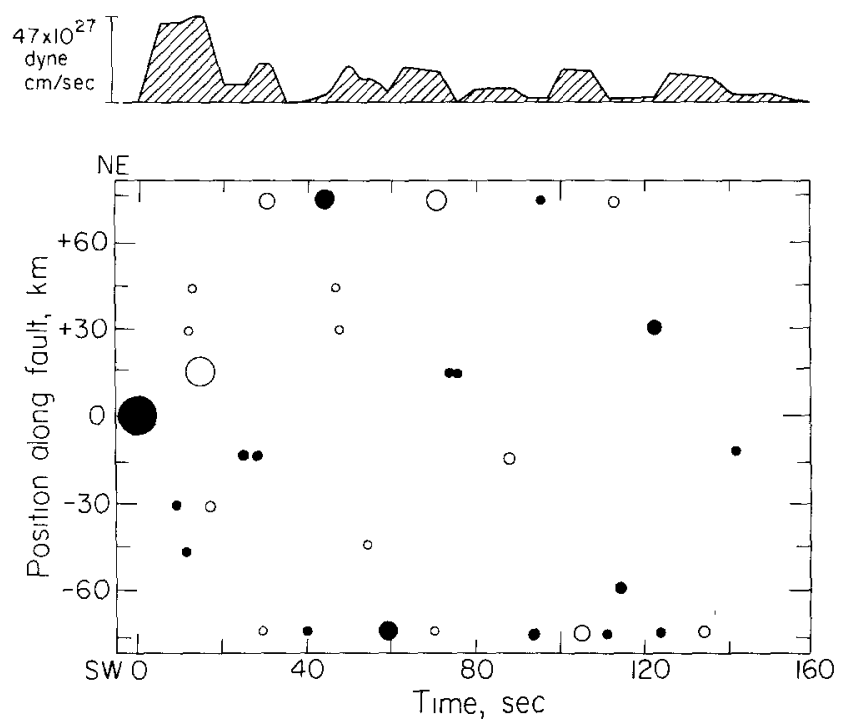

FIG 11. Schematic of the $S H$ simultaneous deconvolution rupture history. The circules indicate location and onset time of large pulses, with filled and open circles showing positive and negative pulses, respectively. Relative pulse moment is roughly given by circle diameter

estimate an average slip of $2.1 \mathrm{~m}$ for this event. The actual fault width to use is uncertain due to the difficulties in constraining the aftershock depths (Smith et al., 1981). The Eureka event clearly represents significant deformation of the Gorda plate, with the fault orientation being consistent with a north-south compressional stress regime.

The stability of the moment estimate over the large range in period from 15 to 512 sec indicates that the Eureka event had no anomalous source spectrum characteristics. Our moment is consistent with the estimate of $1.1 \cdot 10^{27}$ dyne-cm obtained by Dziewonski and Woodhouse (1981) by moment tensor inversion of body and surface waves. The order of magnitude discrepancy in $m_{b}(6.2)$ and $M_{S}$ (7.2) probably reflects the multiple source character of the rupture, extending over a length of about $100 \mathrm{~km}$. 


\section{Conclusions}

The long-period source parameters of the 8 November 1980 Eureka earthquake have been determined using Rayleigh and Love wave data from SRO, ASRO, and IDA stations. The simultaneous moment tensor inversion of this data yields a moment of $1.1 \cdot 10^{27}$ dyne-cm for a left-lateral vertical strike-slip mechanism trending $\mathrm{N} 48.2^{\circ} \mathrm{E}$, with a minor double-couple moment 10 per cent as large as the major double-couple moment. This solution is relatively insensitive to source process time and source depth less than $33 \mathrm{~km}$. Simultaneous inversion of the amplitude data gives a refined moment of $1.3 \cdot 10^{27}$ dyne-cm and strike $\mathrm{N} 49.3^{\circ} \mathrm{E}$. No significant oblique slip is found, although the rake is not well constrained within $\pm 20^{\circ}$. At 256 sec period, no directivity is observed, but associating the northeast-trending nodal plane with the aftershock distribution indicates that the Gorda plate is being internally deformed along northeast-trending structures due to north-south compression. Inversion at other periods confirms the moment and orientation found at $256 \mathrm{sec}$, with no significant variation between 75 and $512 \mathrm{sec}$. The body wave data indicate a multiple rupture with four distinct subevents in the first $80 \mathrm{sec}$ of rupture which give a total moment of $1.3 \cdot 10^{27}$ dyne-cm for the $S H$ waves. The first subevent released $\frac{1}{2}$ the total moment.

\section{ACKNOWLEDGMENTS}

We appreciate helpful suggestions made by Terry Wallace and the anonymous reviewer. Research was supported by the Earth Sciences Section National Science Foundation Grant EAR 78-11973 and by the United States Geological Survey Contract 14-08-0001-19755. One of the authors, T. L., was supported by a National Science Foundation Graduate Fellowshıp.

\section{References}

Driewonski, A. M. and J. H. Woodhouse (1981) Analysis of complex earthquakes, EOS 62, 330.

Eaton, J. P. (1981). Distribution of aftershocks of the November 8, 1980 Eureka earthquake, Earthquake Notes 52, 44-45.

Glbert, F. and A. M. Dziewonski (1975). An application of normal mode theory to the retrieval of structural parameters and source mechanisms from seismic spectra, Phll. Trans. R. Soc. London, Ser. A 278, 187-269.

Kanamori, H. (1970). Velocity and Q of mantle waves. Phys. Earth Planet. Interiors 2, 259-275.

Kanamori, H. (1977). The energy release in great earthquakes, J. Geophys. Res. 82, 2981-2987.

Kanamori, H. and G. S. Stewart (1976) Mode of the strain release along the Gibbs fracture zone, MidAtlantic ridge, Phys. Earth Planet. Interiors 11, 312-332.

Kanamori, H. and G. S. Stewart (1978). Seismological aspects of the Guatemala earthquake of February 4, 1976, J. Geophys. Res 83, 3427-3434.

Kanamor, H. and W. Given (1981). Use of long-period surface waves for fast determination of earthquake source parameters, Phys. Earth Planet. Intertors 27, 8-31

Kikuchi, M. and H. Kanamori (1982). Inversion of complex body waves, Bull. Selsm. Soc. Am. 72, 491506.

McCowan, D. W. and R. T. Lacoss (1978). Transfer functions for the seismic research observatory selsmograph system, Bull. Selsm. Soc. Am. 68, 501-512.

Press, F. (1970). Earth models consistent with geophysical data, Phys. Earth Planet. Interiors 3, 3-22

Silver, E. A. (1971) Tectonics of the Mendocino triple junction, Bull. Geol. Soc. Am 82, 2965-2978.

Smith, S. W (1976). Seismicity north of Cape Mendocino, Selsm Soc. Am. abstracts annual meeting, Edmonton, Alberta Canada.

Smith, S. W., R C. McPherson, and N. I Severy (1981) The Eureka earthquake of 1980, breakup of the Gorda plate, Earthquake Notes 52, 44.

SEISMOLOGICAL LaBoratory

California Institute of Technology

Pasadena, California 91125

Contribution No. 3662

Manuscript recerved 24 July 1981 